Document downloaded from:

http://hdl.handle.net/10251/43821

This paper must be cited as:

Sadiq, AS.; Abu Bakar, K.; Ghafoor, KZ.; Lloret, J.; Khokhar, R. (2013). An Intelligent Vertical Handover Scheme for Audio and Video Streaming in Heterogeneous Vehicular Networks. Mobile Networks and Applications. 18(6):879-895. doi:10.1007/s11036-0130465-8.

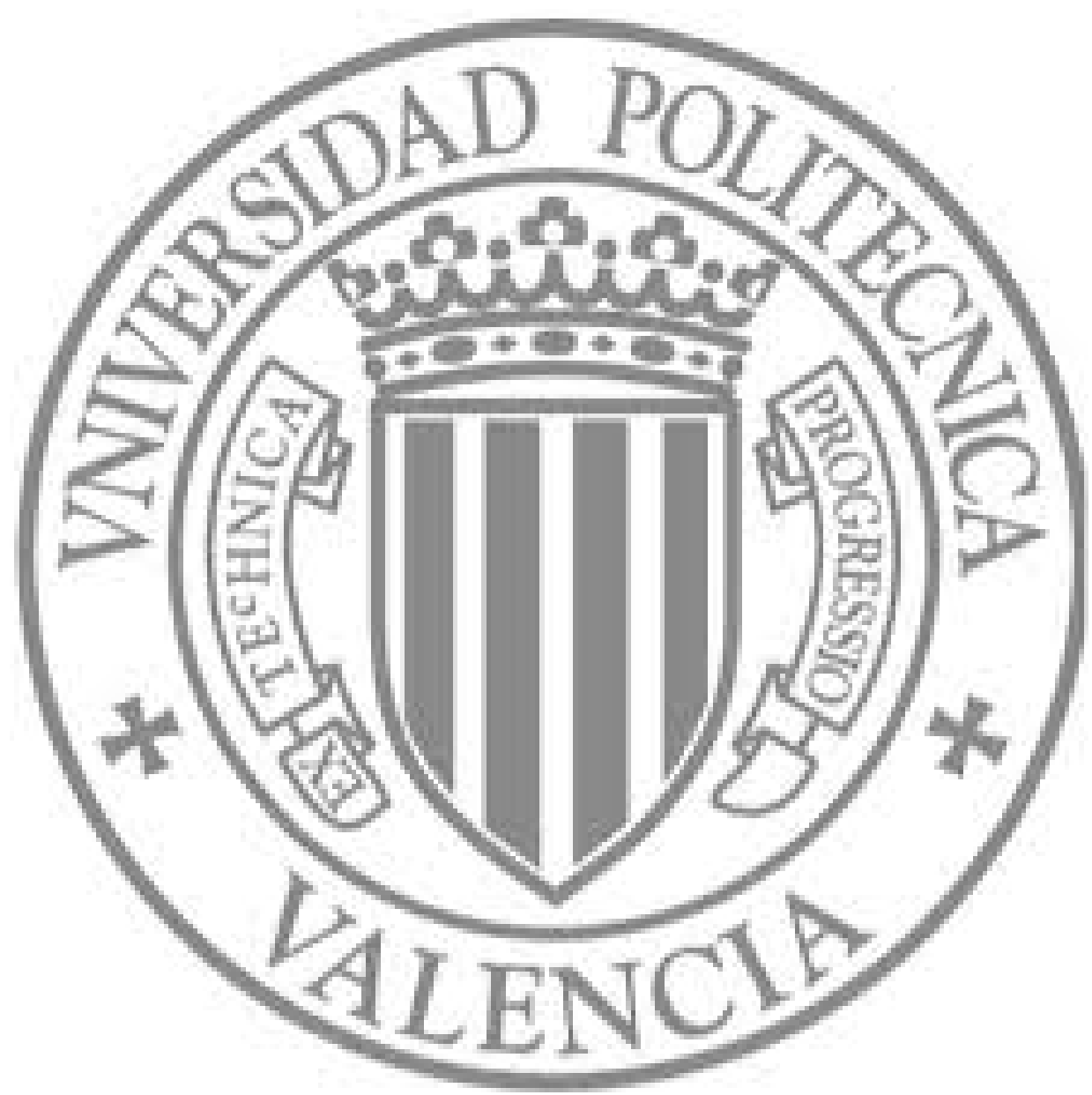

The final publication is available at

http://dx.doi.org/10.1007/s11036-013-0465-8

Copyright Springer Verlag (Germany) 


\title{
An Intelligent Vertical Handover Scheme for Audio and Video Streaming in Heterogeneous Vehicular Networks
}

\author{
Ali Safa Sadiq · Kamalrulnizam Abu Bakar . \\ Kayhan Zrar Ghafoor · Jaime Lloret · Rashid Khokhar
}

the date of receipt and acceptance should be inserted later

\begin{abstract}
In heterogeneous vehicular networks, the most challenging issue is obtaining an efficient vertical handover during the vehicle roaming process. Efficient network selection process can achieve satisfactory Quality-of-Service for ongoing applications. In this paper, we propose an Intelligent Network Selection (INS) scheme based on maximization scoring function to efficiently rank available wireless network candidates. Three input parameters were utilized to develop a maximization scoring function that collected data from each network candidate during the selection process. These parameters are: Faded Signal-to-Noise Ratio, Residual Channel Capacity, and Connection Life Time. The results show that the proposed INS scheme is more efficient at decreasing handover delays, End-to-End delays for VoIP and Video applications, packet loss ratios as well as increasing the efficiency of network selection processes in comparison with the state of the arts.
\end{abstract}

Keywords Heterogeneous Vehicular communication . Efficient Vertical Handover · Intelligent Network Selection · Maximization Scoring Function

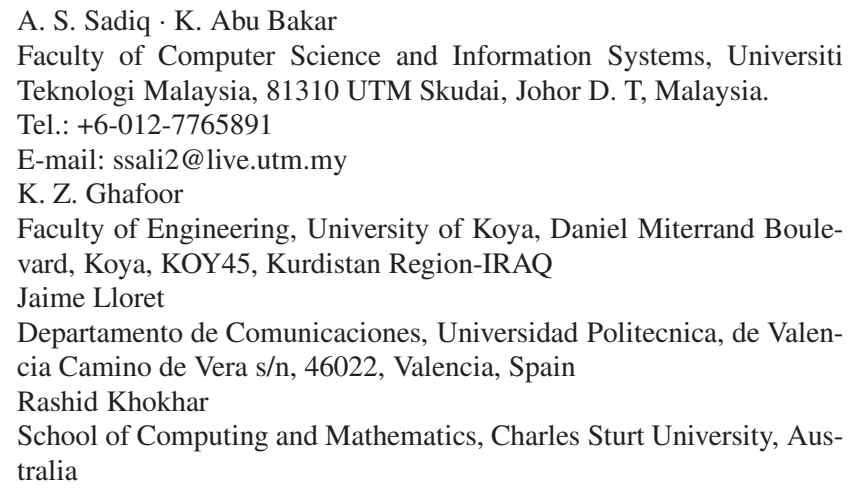

\section{Introduction}

Emerging wireless communication technologies have developed as a way to provide Quality-of-Services (QoS). A variety of wireless technologies are involved in maintaining broadband coverage with high QoS and seamless mobility. In vehicular ad-hoc networks (VANET) two types of communication technologies are involved. One technology is vehiclesto-vehicle (V2V), which typically deals with communication between smart vehicles. The second technology is vehicleto-infrastructure (V2I), which transmits information between a vehicle and fixed infrastructure normally installed on the side of the road. The focus of this paper was on V2I, which is the infrastructure network in the area surrounding VANET and which enables in-vehicle entertainment online applications. In the other words, V2I allows users to access internet service in addition to on-line entertainment applications while they are travelling in their vehicles by using any available 3G, 4G, WiMAX or Wifi hotspots.

The wireless link connection quality in V2I networks faces many obstacles such as poor wireless channel quality and connectivity breaking down due to high travel speeds. As a result, there is a need for efficient vertical handover processes that consider different levels of link quality and the mobility aspects of vehicular networks ( [1], [2], [3], [4] and [5]). A vehicle should be able to select the most appropriate road side network access point in order to maintain the required QoS for ongoing applications. For instance, when a vehicle performs a handover to a network with a low or unstable signal quality due to fading phenomena, the probability of handover failure will be higher. Thus, the handover decision in these cases can be considered to be inefficient because of faded received signals that cause high levels of handover failure.

Additionally, network selection processes should avoid network candidates with low channel bandwidth caused by 
many vehicles associated with a particular network candidate. Besides, the link connection break down probability during a network selection process when a vertical handover decision is made must also be considered. By looking at these factors, an efficient vertical handover can be achieved for heterogeneous V2I networks with the necessary QoS to support running applications [6] and [7].

In this paper an efficient vertical handover was proposed for heterogeneous V2I wireless networks. The handover decisions were achieved by utilizing the proposed Intelligent Network Selection (INS) scheme. The proposed INS scheme performed the developed maximization scoring function in order to rank available wireless network candidates in the area surrounding a VANET. Throughout the proposed INS scheme, vertical handovers between the Universal Mobile Telecommunications System (UMTS), which is a third Generation (3G) mobile cellular system for networks and Wireless Local Area Network (WLAN), were performed efficiently.

The contributions made by this paper include the introduction of an efficient vertical handover decision process between UMTS-to-WLAN and vice versa. This was achieved by developing an intelligent network selection scheme that took into consideration more realistic quality metrics as a way to identify the candidacy scores when electing a particular network. A Faded Signal-to-Noise Ratio metric was calculated by implementing the Rayleigh Fading Model into the radio channel for OMNET++ simulation scenario. Thus, vehicles were allowed to calculate the actual received signal from each wireless access link after considering all possible obstacles and other fading phenomena. Additionally, the residual channel capacity and connection life time of each available network candidate were identified and calculated. Eventually, the proposed INS scheme in this paper will contribute by effectively decreasing the delay associated with handover process in addition to reducing link connection breakdown and unnecessary handovers probabilities.

The remainder of the paper includes the following: Section 2 provides a summary of the literature related to handover systems that may be applied to VANETs, Section 3 presents the design for the proposed INS scheme, Section 4 discusses lookup table developed in this study to find the minimum required channel capacity. The process of the INS Algorithm is presented in Section 5 followed by the results in Section 6 and a conclusion in Section 7.

\section{Related Works}

Several recent studies have discussed vertical handover decision making and network selection processes used to elect the best network candidate for a VANET using V2V communications [8]. Throughout these studies, mathematical theories were developed and utilized for addressing these is- sues. . The authors in [9], discussed the most important mathematical theories used for network selection processes with heterogeneous networks. Moreover, they compared the schemes of various mathematical theories and discussed how to combine mathematical theories. Furthermore, they proposed an integrated scheme utilizing multiple attribute decision making for the network selection process.

The proposed integrated scheme was computationally expensive because many selection metrics should be identified for each network candidate with each decision making attempt. The preparation process conducted before combining the attributes, in addition to the weighting and attribute adjustment procedures, normally resulted in slow decision making. The vertical handover decision was also taken when the first available network for the best candidate was obtained via an integrated scheme when it was better than the current network. This can lead to unnecessary handovers that effect negatively the ongoing session, especially for realtime applications when a network was unable to maintain a real-time session.

On the other hand, the utility theory was analyzed by [10]as a way to identify a suitable vertical handover decision mechanism. In this theory, the Sigmoidal utility function was considered for the network selection process. One of the issues facing the use of this utility function is that the parameters within the sigmoidal function can be different than those of the features of the selected attributes [9]. Moreover, the attributes considered of only the network's bandwidth and the price to be inserted into utility function regardless some other network selection aspects.

In a study conducted by [11], the authors proposed a protocol and algorithm that performed an IPTV handover that used the best available WLAN when the QoS parameters received by the end user reached the selected threshold. This insured that the QoS for the user during the connection period would be maintained. However, this protocol focused on handovers using IEEE 802.11 and did not include any other network standards.

Other studies such as one conducted by [12] and [13] developed vertical handover algorithms for use between a WLAN to a $3 \mathrm{G}$ network and vice versa. When these algorithms were used, the handovers were normally triggered when the vehicle entered the boundary area of the WLAN. The handover procedure was completed before the vehicle left the WLAN coverage area. These algorithms functioned efficiently when a handover from a WLAN to a $3 \mathrm{G}$ network was needed. Besides, they can maintain the handover failure probability from WLAN to $3 \mathrm{G}$ networks. However, the vertical handovers were inefficient when the vehicle moved across an area close to the boundary of the WLAN coverage at speed. In these situations, vertical handover to the WLAN were unnecessary. There are still a few unresolved issues facing these proposed vertical handover algorithms due to 
the waste of network resources that occurred as the result of unnecessary handovers.

\section{Intelligent Network Selection (INS) Scheme mode}

In the proposed INS scheme, three criteria were considered to measure network performance. These criteria were the faded Signal-to-Noise Ratio (SNR), Residual Channel Capacity and Connection Life Time. In order to describe the key challenges for each metric and the way that they were used to extract the status evaluation value, a detailed discussion is presented in the subsections below.

\subsection{Faded Signal-to-Noise Ratio}

SNR is the power ration of a signal divided by the noise power at a particular point in the transmission. The SNR value obtained can be considered to be of high quality when the power of the signal received is more than the power of the noise. The SNR can be affected by different factors, such as vibrations, wind, rain, and temperature. Whenever signal power is equal to or less than noise power, the SNR value is considered to be a low quality value, unable to carry the ongoing session. The SNR value is measured and obtained by a vehicle through the $\mathrm{AP}^{\prime} \mathrm{s}$ beacon frames that are sent every $100 \mathrm{~ms}$.

In order to obtain the SNR input metric range for a WLAN the $S N R_{\text {Range }}$ value was assumed to be from 10 to $50 \mathrm{~dB}$, based on a study conducted by [14]. Thus, in the proposed INS scheme, moving vehicles continuously monitored the SNR to ensure that the SNR level of the current and target networks were in the acceptable level. Thus, by considering the SNR status as an input metric, the QoS of the performing application was ensured.

The faded wireless channel was considered in the proposed INS scheme in order to achieve a high level of accuracy for vertical handover decisions. When vehicles roamed, the signal received from the APs or BSs fluctuated. This was due to either Large-Scale (slow) fading or Small-Scale (fast) fading [15]. Slow fading is the average signal power lost due to movement over large areas. In the other words, it can be defined as the received signal variation due to a vehicle's movement away from the transmitter. In order to obtain the WLAN coverage area a log-distance-path-loss model was used in the physical layer of IEEE 802.11 of simulated scenario.

A Rayleigh or Rician random variable distribution was used to model fast fading in the proposed INS scheme. Fast fading occurs when a signal travels from the transmitter to the vehicle over multiple paths caused by propagation mechanisms. In the proposed INS scheme when the network access point had a good SNR value it contributed to increasing the cost score of a candidate AP that would be elected as a next recommended attachment link.

A probability density function was utilized to calculate the SNR received by a vehicle or the transmission range of each AP. The Rayleigh Fading Distribution [15] the probability of times that the received or transmitted signal changed due to the effects of fast fading. Eventually, all of the faded SNRs were processed using a log-distance path loss model for slow fading and a Rayleigh Fading Model for fast fading. The faded SNR was inserted into the proposed INS scheme as a way to extract the quality score for each particular network candidate.

\subsection{Residual Channel-Capacity}

Channel capacity can be represented by bandwidth and it is defined as a remainder of frequency space for a mobile node [16]. For instance, the channel band size of IEEE 802.11 is $20 \mathrm{MHz}$, which reflects the $20 \mathrm{MHz}$ total bandwidth provided by each channel. On the other hand, UMTS is based on cellular networks, which have an allocation of $25 \mathrm{MHz}$ total bandwidth in the $900 \mathrm{MHz}$ frequency range [17]. The average variation for the residual capacity of wireless channels depended on the time that a vehicle remained in each WLAN or UMTS network during its roaming process.

In WLANs, the AP uses Network Allocation Vector (NAV) [18] to infer the status of wireless channel. In BBS, APs send beacon frames containing a CF Parameter Set information element. This frame can be also received by vehicles that are not associated with the BSS. When a NAV counts down to zero, a vehicle is able to send or receive data frames via the Wireless Medium (WM) that is represented as (old NAV).

Before vehicles receive a beacon frame containing a Contention Function $(\mathrm{CF})$ parameter set element, their NAVs will normally be set to the CFPMaxDuration value at the nominal start time of each CFP. The initial NAV's time in our proposed INS scheme was set to the modified parameter called $c f p_{\text {length }}$. This variable contains the default length in seconds of the CFP. When vehicle receives the beacon frame from an AP, the $N A V_{\text {Duration }}$ will be calculated by each vehicle in the way to synchronize their NAV timer; that each vehicle can could use the WM without conflict. The process of updating $N A V_{\text {Duration }}$ was tackle by a vehicle at the beginning of each CFP after they received the NAV settings via beacon frames for the vehicles under $\mathrm{CP}$.

Figure 1a shows that the CF Parameter Set element format contains a set of parameters that are required to support the PCF procedure. The information field in the $\mathrm{CF}$ format consists of 6 octets that distribute the CFPCount, CF PPeriod, CF PMaxDuration, and CF PDurRemaining fields. From CF PMaxDuration, a vehicle can obtain the maximum duration in Time Unit (TU) microseconds, which are inserted into $c f p_{\text {length }}$. Whereas, CFPDurRemaining refers to 


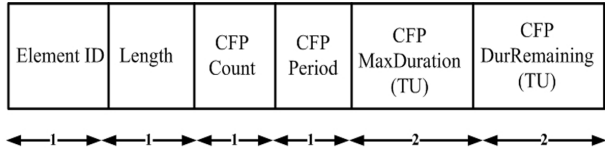

(a) The CF Parameter Set element format [18].

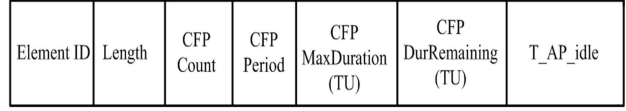

Octets:

(b) The modified CF Parameter Set element with $T_{A P_{\text {idle }}}[18]$.

Fig. 1: Standard and Modified CF Set Element.

the time remaining in the CFP. This value is normally used by vehicles to update their NAVs during CFPs. When anyvehicle, regardless of its association with a BBS, receives the CF Parameter Set information, they will set their NAVs to the CF PMaxDuration. Vehicles will use CF PDurRemaining to update their NAVs during the CF process.

Residual channel capacity can be calculated by determining the average time between the last moment the AP was idle to the moment it became busy and then multiplying this by the elaborated transmission data rate. In the proposed INS scheme, time indicator $T_{A P_{\text {idle }}}$ was modified in order to capture the last time that the AP was idle, represented as a CFP. The modified CF Parameter Set included $T_{A P_{i d l e}}$ was that periodically sent by $\mathrm{AP}^{\prime} \mathrm{s}$ beacon frames as shown in Figure 1b.

The residual channel capacity $R_{C C}$ can be obtained for each AP in the vehicle's scanning range using Formula 1. The total time that an AP was busy from the last monitored period after CFP is represented by $T_{A P_{B u s y}}$. $T_{A P_{\text {idle }}}$ is the last time, measured in seconds, that the AP was idle. The Transmission Data Rate is equal to $11 \mathrm{Mbps}$ for WLANs based on IEEE $802.11 \mathrm{~b}$ standards [18]; which represent the total channel capacity (11Mbps). $T_{A P_{B u s y}}$ can be divided into sub time periods that indicate when the AP was busy. During $T_{A P_{B u s y}}$,vehicle should keep waiting before they are able to use the $\mathrm{AP}^{\prime} \mathrm{s}$ channel after this time has expired. Formula 2illustrates the summation process for all the busy time periods in a WLAN. Where $T_{c p}$ is the busy time period due to $\mathrm{CP}$ for the AP, $T_{p i f s}$ is the time interval utilized in $P C F$ to assign priority access to the $\mathrm{AP}^{\prime}$ s channel by vehicles after $\mathrm{CP}$, and $T_{\text {Ack }}$ ifs is the time delay normally experienced by vehicles to gain PIFS acknowledgement from the PC (AP).

$R_{C C}=\frac{T_{A P_{i d l e}}}{T_{A P_{B u s y}}} \times$ Transmission Data Rate

$$
\begin{array}{r}
T_{A P_{\text {Busy }}}=T_{c p}+T_{\text {pifs }}+T_{\text {Ack }} \text { pifs } \\
T_{\text {total media access delay }}+ \\
+T_{\text {Accuracy-Recovery }}
\end{array}
$$

$T_{p i f s}$ can be divided into $T_{\text {sifs }}$, which is the time interval between each transmitted frame during the CF process, $T_{\text {Ack }}$ ifs is the time delay before the acknowledgement of SIFS from PC (AP) was obtained, and $T_{\text {Slot }}$ is the time slot added in order to calculate $T_{p i f s}$ in $P C F$. In our proposed INS scheme, the $T_{\text {sifs }}$ value was set to $(0.028 \mathrm{~ms})$ and $T_{\text {Slot }}$ was equal to $(0.05 \mathrm{~ms})$. Formula 3 was used to calculate the $T_{p i f s}$ value. $T_{\text {Accuracy-Recovery }}$ is a precision sensitive computation considered in the calculation process of $T_{A P_{B u s y}}$, which was equal to $1 \mathrm{ps}$.

$T_{p i f s}=T_{\text {sifs }}+T_{A c k_{s i f s}}+T_{\text {Slot }}$

In order to accurately calculate the residual channel capacity, MAC delay $T_{M A C_{\text {delay }}}$ was considered to be one of the delay time contributors for $T_{A P_{B u s v}}$. A Contrary, Formula 4

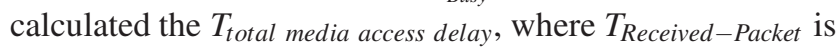
the arrival time of the packet that is currently tackled by the $P C F$ and $T_{\text {Packet-Sent }}$ is the time that the packet was sent to $P C F$.

During the proposed INS scheme process time, $T_{\text {total media access delay }}$ between each data fragments at the end of Media Access Duration was updated as a way to keep track of changes in $T_{\text {total media access delay }}$ and regularly update the entries to Formula 2 to evaluate the $T_{A P_{\text {Busy }}}$.

$T_{\text {total media access delay }}=T_{\text {Received-Packet }}-T_{\text {Packet-Sent }}$

In UMTS based cellular networks, a vehicle receives channel system information via the Broadcast Control Channel $(\mathrm{BCCH})$ during the radio recourse management procedures [17] . The RR-connection setup and service request phases in UMTS networks are controlled by BS of each cell. In this paper, it was assumed that each vehicle received a fixed channel capacity represented by a maximum data rate of $2048 \mathrm{kbit} / \mathrm{s}$ at $10 \mathrm{~km} / \mathrm{h}$ and $384 \mathrm{kbit} / \mathrm{s}$ at $120 \mathrm{~km} / \mathrm{h} \mathrm{[17].}$ For this reason, the residual channel capacity $R_{C C}$ for each UMTS network is equal to the total utilized data rate. This is because a vehicle received the entire $\mathrm{BS}^{\prime}$ s channel capacity during its allocated access time based on a Wideband CodeDivision Multiple-Access (W-CDMA) process [17].

Figure 2 shows that when vehicle numbers decreased it contributed to an increase in the $R_{C C}$. In other words, when the number of vehicles associated with one particular AP was low, this was reflected as $T_{A P_{B u s v}}$. As a result, a higher $R_{C C}$ value was achieved, which indicated that this particular AP was better able to tackle handover processes with low time delay. 


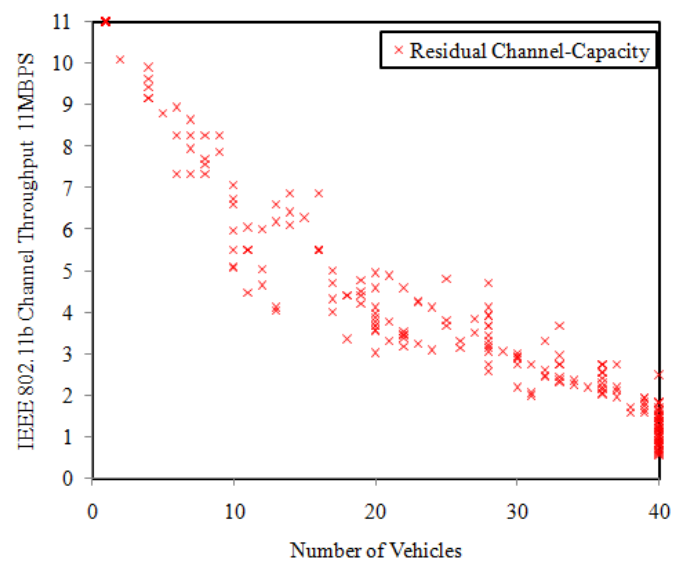

Fig. 2: The variation of Residual Channel-Capacity with Changes in Number of vehicles.

\subsection{Connection Life Time}

In order to decrease the probability of a link connection breakdown, a vehicle must make intelligent handover decisions and avoid unnecessary handover decisions. In order to act intelligently, the connection life time of an AP must be considered as a way to minimize unnecessary handovers from UMTS to WLAN. When a vehicle connects to a UMTS network, the connection life time is normally longer than when it connects to a WLAN because UMTS's coverage area is larger. One cell of UMTS networks can provides up to $5 \mathrm{~km}$ of coverage [19] which allows a vehicle to connect for longer times, compared to a WLAN's that only provided coverage over hundreds of meters [18]. In our proposed INS scheme, the connection life time for each AP was calculated to avoid unnecessary WLAN handovers.

Figure 3 illustrates a scenario in which a vehicle is moving towards a Wifi's AP, which is currently connected to UMTS's BS1. The vehicle will reach the WLAN's coverage area boundaries at time period point (time starts WLAN coverage area $\left.T_{S_{W L A N}}\right)$. In this paper, it was assumed that each AP in the WLANs covered a circular area with a fixed transmission radius. This assumption was based on the transmission power used for each AP found in the physical layer settings of WLANs. Therefore, the distance between each of the starting boundary's points and the opposite points are the same.

As it depicted in Figure 3, when a vehicle enters the AP coverage area at $T_{S_{W L A N}}$ with a certain velocity vector, the point that the vehicle leaves this AP at time period point will be the end of the WLAN coverage area $T_{E_{W L A N}}$. The time between $T_{S_{W L A N}}$ and $T_{E_{W L A N}}$ is identified as the WLAN connection life time $\left(T_{C W L A N}\right)$. This $T_{C W L A N}$, which normally changes based on the distance between a vehicle's current position vector (Moving Point) and the $\mathrm{AP}^{\prime}$ 's position (Fixed Point) with respect to the current vehicle's velocity. The AP's

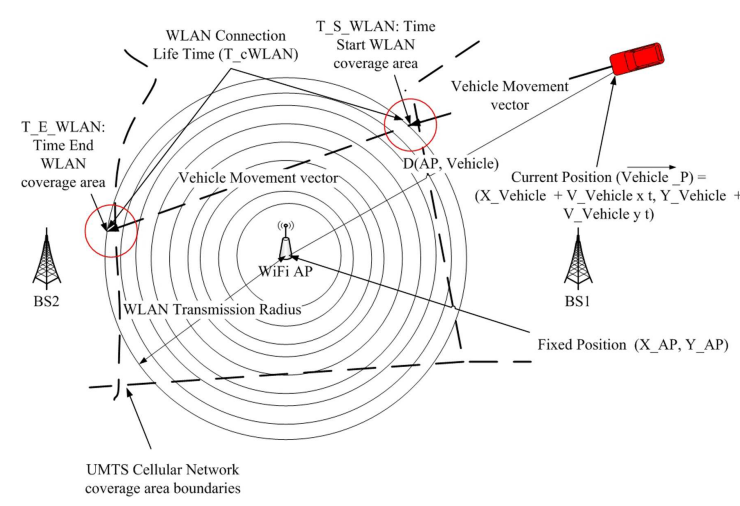

Fig. 3: Vertical handover scenario from UMTS to WLAN based on connection life time calculation.

position is initially identified as $\left(X_{A P}, Y_{A P}\right)$, whereas the vehicle's current position vector can be identified using Formula 5

Vehicle $_{\mathbf{P}}=\left(X_{\text {vehicle }}+V_{\text {vehicle }_{x}} t, Y_{\text {vehicle }}+V_{\text {vehicle }_{y}} t\right)$

where $X_{\text {vehicle }}, Y_{\text {vehicle }}$ are the current $\mathrm{x}$ and $\mathrm{y}$ axis of vehicle's position, and $\mathbf{V}_{\text {vehicle }_{\mathbf{x}}}, \mathbf{V}_{\text {vehicle }_{\mathbf{y}}}$ are the velocity vectors of each $\mathrm{x}$ and $\mathrm{y}$ axis, respectively. The vehicles position vector at $t$ is calculated using the time monitored beginning from when the vehicle began collecting the $\mathrm{AP}^{\prime}$ s RSS until the current time.

In order to calculate the distance square $\partial^{2}(t)$ between vehicles and every AP in scanning range, Formula 6 was used. Where vehicle $\mathbf{P}_{\mathbf{x}}$ is the vehicle's position vector on the $\mathrm{x}$-axis, $A P_{P_{x}}$ is the $\mathrm{AP}^{\prime} \mathrm{s}$ position on the $\mathrm{x}$-axis, vehicle $\mathbf{P}_{\mathbf{y}}$ is the vehicle's position vector on the y-axis, and $A P_{P_{y}}$ is the $\mathrm{AP}^{\prime} \mathrm{s}$ position on the $\mathrm{y}$-axis:

$\partial^{2}(t)=\left(\text { vehicle }_{\mathbf{P}_{\mathbf{x}}}-A P_{P_{x}}\right)^{2}+\left(\text { vehicle }_{\mathbf{P}_{\mathbf{y}}}-A P_{P_{y}}\right)^{2}$

Formula 7, shows the $\partial^{2}(t)$ when Formula 5 is substituted for Formula 6 in order to illustrate the calculation process of distance square between each vehicle and an AP. Assume that $\gamma_{x}=\left(X_{\text {vehicle }}-X_{A P}\right)$ and $\gamma_{y}=\left(Y_{\text {vehicle }}-Y_{A P}\right)$, when these values are used in Formula 7, a simplified form of $\partial^{2}(t)$ results as shown below in Formula 8:

$$
\begin{array}{r}
\left.\partial^{2}(t)=\left[\left(X_{\text {vehicle }}-X_{A P}\right)+V_{\text {vehicle }_{x}} t\right)\right]^{2} \\
+\left[\left(Y_{\text {vehicle }}-Y_{A P}\right)+V_{\text {vehicle }_{y}} t\right]^{2} \\
\partial^{2}(t)=\left(\gamma_{x}^{2}+\gamma_{y}^{2}\right)+t^{2}\left(V_{\text {vehicle }_{x}}^{2}+\right. \\
\left.V_{\text {vehicle }_{y}}^{2}\right)+2 t\left(\gamma_{x} V_{\text {vehicle }_{x}}+\gamma_{y} V_{\text {vehicle }_{y}}\right)
\end{array}
$$


Any values resulting from Formula 8,are positive. Hence, the lowest value of $\partial^{2}(t)$ can be achieved only when the derivative $\partial^{2^{\prime}}(t)=0$. The value of $t$ can be calculated as:

$\bar{t}=\frac{\left(\gamma_{x} V_{\text {vehicle }_{x}}+\gamma_{y} V_{\text {vehicle }_{y}}\right)}{\left(V_{\text {vehicle }_{x}}^{2}+V_{\text {vehicle }_{y}}^{2}\right)}$

Formula 9 provides two values for the connection life time between each vehicle and an AP. When the $\bar{t}$ value is positive, it indicates that the vehicle is facing the same direction as the AP or is moving towards the AP. However, when the value is negative, it infers that the vehicle is moving away from or facing away from the AP. Thus, the resulting connection life time of each AP in scanning range will be inserted into the proposed INS scheme in order to collaborate with the other two metrics (FSNR and Residual Channel capacity) to achieve a high level of accuracy for ranking the quality cost of each available network candidate.

\subsection{Utilized Maximization Scoring Function}

After the three input metrics of INS scheme were identified, an aggregating function was used to combine all the network selection criteria into a single function INS that was then used to elect the best candidate network. This score function was a single ranking measure that combined all the aforementioned metrics into a single metric. For instance, a score function INS is based on the $j$ network selection metrics $\eta_{i}=\left\{\eta_{i 1}, \eta_{i 2}, \eta_{i 3}, \ldots, \eta_{i j}\right\}$ and each of the candidate networks $n_{i}$ has numerical values in the range of $\left[\eta_{i}^{\text {min }}\right.$ to $\left.\eta_{i}^{\text {max }}\right]$. Thus, a multi-metric scoring function can be expressed as follows [20]:

$$
f\left(\eta_{i 1}, \eta_{i 2}, \eta_{i 3}, \ldots, \eta_{i j}\right)=X \times \eta_{i 1}^{\sigma_{1}} \times \eta_{i 2}^{\sigma_{2}} \times \eta_{i 3}^{\sigma_{3}} \ldots . . e t a_{i j}^{\sigma_{j}}+Y_{\max }
$$

Where $Y_{\max }$ is the maximum value of the multi criteria function $f\left(\eta_{i 1}, \eta_{i 2}, \eta_{i 3}, \ldots, \eta_{i j}\right), X$ is the variable dependent weights of the limiting condition, and $\left(\sigma_{1}, \sigma_{2}, \sigma_{3} \ldots, \sigma_{j}\right)$ is a $\mathrm{j}$-weight array used to assign priority to the handover decision making metrics. For example, the network criteria metric with a higher weight factor contributes more to the network election process. In the proposed INS scheme, three input metrics were utilized to make handover decision between UMTS networks and WLANs. Thus, the network selection value was calculated as follows:

$$
\begin{array}{r}
f\left(F S N R_{i}, R_{(c c) i}, t_{(\text {connection }) i}\right)=X \times F S N R_{i}^{\sigma_{1}} \\
\times R_{c c_{i}}^{\sigma_{2}} \times t_{\text {connection }_{i}}^{\sigma_{3}}+Y_{\text {max }}
\end{array}
$$

The maximum value of $f\left(F S N R_{i}, R_{(c c) i}, t_{(\text {connection }) i}\right)$ occurs when its derivative is equal to zero. As a result, the value of $\mathrm{X}$ is given as:

$X=\frac{-Y_{\max }}{F S N R_{\text {max }}^{\sigma_{1}} \times R_{(c c)_{\max }^{\sigma_{2}}}^{\sigma_{2}} \times t_{(\text {connection })_{\max }}^{\sigma_{3}}}$

For instance, when the obtained cost value of three input metrics $f\left(F S N R_{i}, R_{(c c)_{i}}, t_{(\text {connection })_{i}}\right)$ for one AP candidate belongs to WLAN equals to 0 , means the handover is highly recommended with that particular WLAN. On the other hand, if the values obtain by using Formula 11 was equal to 1 , then the handover is not preferred for this AP candidate. Thus, the $X$ value can be calculated by using Formula 12, where $Y_{\max }=1$ (the maximum number can be achieved via this formula in the range between 0 to 1 ), maximum FSNR value is $50 \mathrm{~dB}$, Maximum $R_{(c c)}$ is 1 , Maximum $t_{\text {(connection) }}$ equals to 1 . Therefore, when $\sigma_{1}=0.9, \sigma_{2}=0.2, \sigma_{3}=0.049$ with the aforementioned maximum metrics' values and when apply Formula 12 as $X=\frac{-1}{50^{0.9} \times 1^{0.2} \times 1^{0.049}}$ which results $X=$ -0.0296 .

In order to illustrate a practical example of INS scheme's calculation for network election process, when the obtained FSNR value is $32 \mathrm{~dB}, R_{(c c)}$ is 0.8 and $t_{\text {(connection) }}$ is 0.01 by utilizing Formula 9 the vertical handover cost with WLAN is $(0.7365)$. The obtained cost value indicates that, the vertical handover with WLAN is not preferable since the cost of handover is high. This due to the fact that, when $t_{\text {(connection) }}$ is low, the handover with WLAN is highly not recommended regardless to the values of each FSNR and $R_{(c c)}$. Thus, the INS scheme can prevent vehicles from obtaining unnecessary vertical handovers with WLAN by achieving a cost number by elaborating the maximization cost function in the network candidate election process.

Figures $4 \mathrm{a}$ and $4 \mathrm{~b}$,illustrate the correlation between the three input metrics and the resulting vertical handover cost. Figure 4a reveals that, the cost was 0 (highvalue) when both $F S N R$ and $t_{\text {(connection) }}$ reached maximum value. When $t_{\text {(connection) }}$ or FSNR values decreased, the cost function fell and was close to the lowestvalue, 1cost. The same concept is shown in Figure $4 \mathrm{~b}$ where the correlation between $t_{\text {(connection) }}, R_{(c c)}$ and the cost of vertical handovers are depicted.

\section{Lookup Table of Minimum Required Channel Capacity for Real-Time Applications}

In the proposed INS scheme, when a vehicle was in busymode and the real-time applications were running, the minimum required channel capacity for running application $\left(a p p_{c_{i}}\right)$ was identified. The reason behind this process is that realtime applications are susceptible to QoS degradation due to increasing packet loss ratios that are caused by handover 


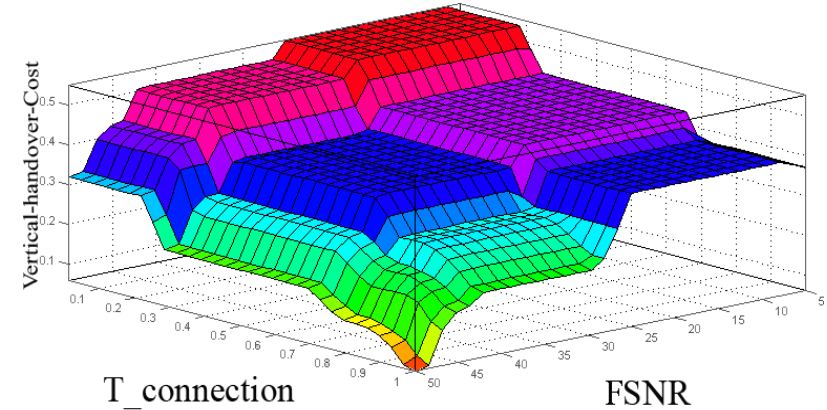

(a) The correlation between $t_{\text {(connection) }}$, FSNR and vertical handover cost values.

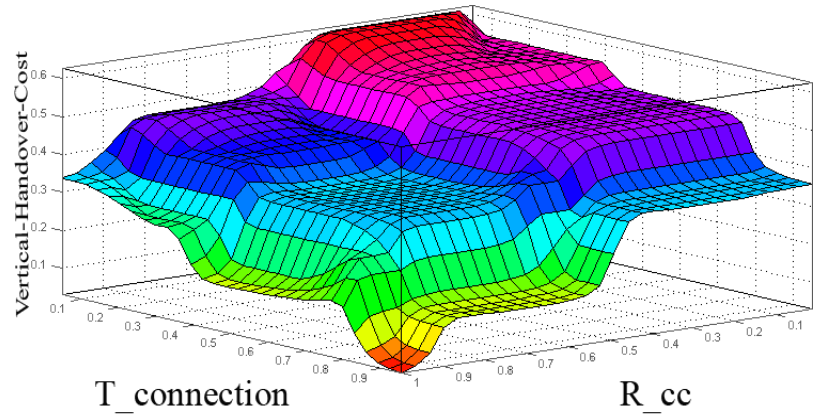

(b) The correlation between $t_{(\text {connection })}, R_{(c c)}$ and vertical handover cost values.

Fig. 4: Analyses of the Proposed Maximization Scoring Function.

delays. Moderate wireless channel's data rates are suitable for voice and low quality video applications [21]. Hence, in the proposed INS scheme when a current session is a real-time application (VoIP or Video), the current residual channel capacity $\left(C R_{c}\right)$ for the current network connection (Cnet) was compared to the app $_{c_{i}}$ of current application $_{i}$. If $C$ net obtained $C R_{c}$ greater or equal to $a p p_{c_{i}}$, then the vehicle remained connected to Cnet as a way to avoid any unnecessary handover decisions. By using the proposed INS scheme, real-time applications avoided unwanted delays caused by handover processes since Cnet provided sufficient channel capacity to maintain these applications.

A lookup table was developed for use by the INS algorithm as a way to define the $a p p_{c_{i}}$ for each of VoIP and Video real-time applications. In order to more precisely identify the minimum acceptable channel capacity, a simulator based scenario was conducted to recognize the minimum level for real-time applications. One AP based on IEEE 802.11b standard with a transmission power of $14 \mathrm{dBm}$ was assigned to cover the area between 380 and 449 meters with Wifi service that was used as a WLAN. In addition, a vehicle and one fixed server were used in the simulated area to generate real-time traffic. VoIP and video traffic was tested separately using three different AP data rates at particular times during the simulator run. Thus, the QoS for VoIP and video traffic was measured each time the AP data rate was changed as a way to monitor the minimum acceptable data rate for VoIP and video traffic $\left(a p p_{c_{i}}\right)$.

VoIP with Codec [G.729A (CS-ACELP)] [22] is used alone under IEEE $802.11 \mathrm{~b}$ AP with $2 \mathrm{Mbps}$, 4Mbps and $11 \mathrm{Mbp}$ data rates. In order to establish $a p p_{c_{i}}$ of VoIP traffic, the QoS verified the three data rates. Figure 5 shows the VoIP evaluation based on two verification metrics, Packet End - to End Delay and Mean Opinion Score (MOS), which were monitored during the simulation [23]; [24]. The QoS measurement results for the VoIP with Codec G.729A for all three data rates are illustrated in Figure 5a.
The total End-to-End Delay was measured by the seconds that it took the VoIP call with a simulated time of 200 seconds as depicted in Figure 5a. Based on the settings for the simulation, the voice call started after 111.6 seconds and ended at 178.2 seconds. The results in Figure 5areveal that the VoIP (G.729A) call placed with a data rate of $2 \mathrm{Mbps}$ had the highest End-to-End Delay, which fluctuated between 0.1 to 0.11 seconds. Whereas the End-to-End delay with AP maintains data rate of $4 \mathrm{Mbps}$ was constantly equals to 0.085 sec. On the other hand, when the elaborated data rate was $11 \mathrm{Mbps}$, the Packet End-to-End delay decreased until if fell between 0.064 and 0.073 seconds.

[24] mentioned that delay limits for one-way transmission according to the Telecommunication Standardization Sector ITU-T Rec. G.114 for End-to-End Delays was in the range of $0-0.15$ seconds. Using these standards, the VoIP call discussed above would be acceptable to most users. However, [25] suggested that the maximum one-way delay should be 0.075 seconds for VoIP traffic in the WLAN. Thus, the results shown in Figure 5a reveal that the Packet End-to-End Delay for a data rate of $4 \mathrm{Mbps}$ was below 0.15 seconds indicating that a data rate of $2 \mathrm{Mbps}$ can provide QoS for VoIP traffic's with acceptable delays.

The MOS value was collected separately during the VoIP call session for each data rate. Figure 5bshows the MOS values during the simulated time for the VoIP (G.729A) call processed using three different data rates (2Mbps, $4 \mathrm{Mbps}$ and $11 \mathrm{Mbps}$ ). As defined in a study conducted by [26], the MOS scoring value should range 1-5 where 1 indicates unsatisfactory speech quality and 5 indicates excellent speech quality. As shown in Figure 5b, when the VoIP call used a $11 \mathrm{Mbps}$ data rate, the MOS value fluctuated between 3.6 to 3.7 indicating that the voice quality was good. When the data rate changed to $4 \mathrm{Mbps}$, the MOS value fell to 3 (fair quality). Finally, the MOS value when the data rate was $2 \mathrm{Mbps}$ was in the range of 2.4 to 2.5 during VoIP call and the voice quality was considered to be poor. 


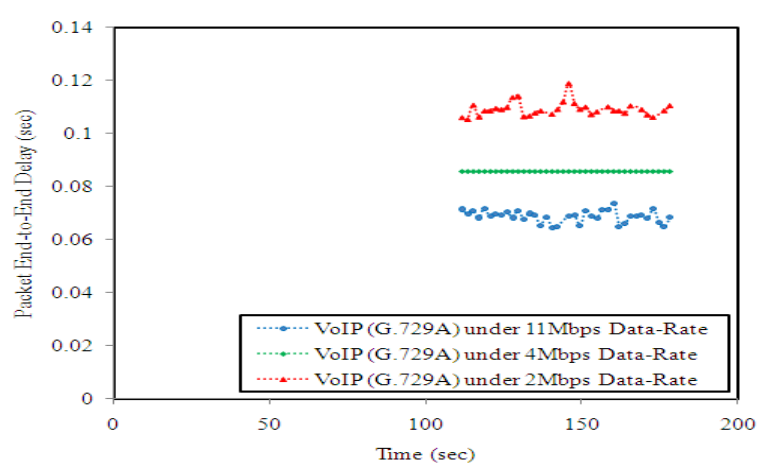

(a) Packet End-to-End Delay.

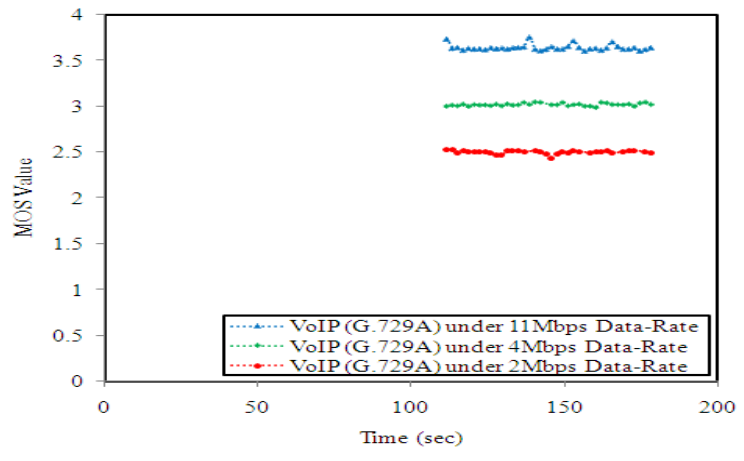

(b) The Mean Opinion Score.

Fig. 5: Evaluation VoIP under IEEE $802.11 \mathrm{~b}$ AP of $2 \mathrm{Mbps}$, 4Mbps and 11Mbps data rates.

The second entry in the lookup table was the acceptable wireless channel capacity for video applications. In order to identify the minimum required channel capacity to maintain an acceptable video streaming session, the same simulator scenario used with VoIP was used for a MPEG-2 video session. MPEG-2 Phase Alternating Line (PAL) format, CIF/SIF (625 lines) with frame size of $352 * 288$ pixels and frame rate of 25 frames per second, was generated between a vehicle and a server and it acted as a video destination. Three different data rates were used with IEEE $802.11 \mathrm{~b}$ AP. At each interval, one of the data rates was used and the Packet End-to-End Delay for the Video MPEG-2 was captured as a way to illustrate the delay variations with respect to changing channel capacities.

The Packet End-to-End Delay that occurred with video applications can be defined as the time from when a packet is sent out by a video calling party to the time the packet reaches the receiving party. Figure 6 shows the Packet Endto-End Delay obtained during the simulation time for each of the three data rates. When the data rate was equal to $11 \mathrm{Mbps}$, the Packet End-to-End Delay stayed in the range of 0.063 seconds during the time the video traffic was receiving. This time delay was considered to be acceptable level and it allowed the Video MPEG-2 traffic to be received with low packet loss ratio as proposed by [27] and [22].

When the data rate decreased to $6 \mathrm{Mbps}$, the Packet Endto-End Delay increased slightly to 0.083 seconds during the video receiving period. The delay with $6 \mathrm{Mbps}$ data rate was still below $100 \mathrm{~ms}(0.1 \mathrm{sec})$ during the video session. A Packet End-to-End Delay of $100 \mathrm{~ms}$ was defined as the maximum acceptable delay for video applications by [27] and [22].

The Packet End-to-End Delay exceeded the acceptable delay $(100 \mathrm{~ms})$ when the data rate decreased to $2 \mathrm{Mbps}$. More precisely, at the 106.2 seconds when the video MPEG-2 was received by the vehicle, the Packet End-to-End Delay was 0.151 seconds. After 108 seconds the delay increasing sharply to 0.352 seconds and kept increasing until it reached 0.489

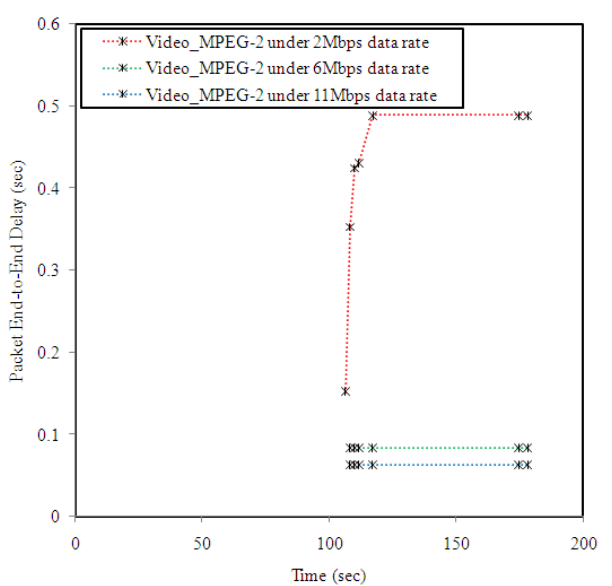

Fig. 6: The variation in Packet End-to-End Delay of Video (MPEG-2) under IEEE $802.11 \mathrm{~b}$ AP of 2Mbps, 6Mbps and $11 \mathrm{Mbps}$ data rates .

seconds. This delay while using the $2 \mathrm{Mbps}$ data rate was considered to be an unacceptable delay and the video session could not be recovered due to high packet loss ratio [27] and [22].

Using the developed lookup table, the channel for the current network Cnet is moderated in terms of its residual capacity so that it can be compared to the values related to each particular application in this table. Therefore, the minimum channel capacity (data rate) for VoIP Codec [G.729A (CS-ACELP)] applications is 4Mbps. Whereas, Video (MPEG2 CIF/SIF (625) $352 * 288$ pixels) has a minimum channel capacity (data rate) of $6 \mathrm{Mbps}$. The reason behind selecting a higher minimum channel capacity for video applications is that video traffic is considered to be a heavy traffic with high numbers of frame sequences (I, P and B frames) [27].A higher data rate for video applications is required. 


\section{INS Algorithm Process for Vertical Handover Decision-Making}

In order to identify and select the most qualified network candidate as the next wireless access point, each vehicle executed the INS algorithm in the network access area. Figure 7 provides a flow chart for the proposed INS scheme for handover decision making. Firstly, each vehicle was assumed to be aware of its own current associated network $\left(\right.$ net $\left._{i}\right)$ ID and its cost $\left(C r_{c_{i}}\right)$ based on previous INS execution. Thus, each iteration of the INS algorithm reads the $n e t_{i}$ and $\mathrm{Cr}_{c_{i}}$ for the current associated network.

The two main wireless interfaces, including WLAN were regularly checked by the proposed INS algorithm. When $n e t_{i}$ was a WLAN, the vehicle's status was first identified to determine if it was in idle or busy mode. When a vehicle was in idle-mode, which was determined by the parameter idle, the INS algorithm selected the network with $\operatorname{Max}_{c_{i}}$ using the MaxNetworkCost Procedure. The handover with this network was initiated without considering any other network or mobility aspects because, in idle - mode a vehicle does not run any applications. Thus, any handover decisions that previously may have been processed by vehicle even if they were unnecessary did not negatively reflect the performance of the network. Afterwards, when a handover already performed the net $_{I D}, \operatorname{Max}_{c_{i}}$ and $R_{c_{i}}$ for the selected new network, the new network will use the parameters of the current network as mentioned in the INS algorithm.

When a vehicle is in busy-mode, its wireless channel can execute various real time and non-real time applications. For instance, VoIP and Video conferencing are considered to be real-time applications due to their sensitivity to packet latency. Whereas, FTP and HTTP refer to data applications that are normally used to transfer data and information over wireless mediums and are considered to be non-real time applications.

The continuity of on-line sessions has a higher priority than the accuracy of the received data in real time applications. Therefore, the link connection delay is considered to be a crucial parameter for delay sensitive applications. Thus, unnecessary handover decision must be avoided when performing real-time applications. Additionally, when data transfer applications run during the busy-mode, the accuracy of transfer data is also important.

In order to avoid any unnecessary handover decisions during real-time sessions, the current application should be identified as being either a real or non-real time application. The Video $o_{\text {Port }}$ and VoIP Port parameters were used to identify the port number for Video and VoIP applications, respectively. The port number for a current application ${ }_{i}$ used in the transport layer was (application $i_{\text {port }}$ ). During busy-mode and each time a handover decision was needed, application $_{i_{p o r t}}$ compared and identified (Video Port and VoIP Port) to deter-
Table 1: Abbreviations and Symbols of INS Algorithm and MaxNetworkCost Procedure

\begin{tabular}{|c|c|}
\hline $\begin{array}{l}\text { Abbreviations and } \\
\text { Symbols }\end{array}$ & Notations \\
\hline$V_{i}$ & The velocity of vehicle $e_{i}$ \\
\hline net $_{i}$ & $\begin{array}{l}\text { The available networks number in scanning } \\
\text { range(UMTS and WLAN) }\end{array}$ \\
\hline Cnet & $\begin{array}{l}\text { The current associated network } \text { net }_{i} \text { ID } \\
\text { (UMTS or WLAN) }\end{array}$ \\
\hline $\operatorname{Max}_{c_{i}}$ & Maximum $C_{i}$ of $n e t_{i}$ \\
\hline$n e t_{I D}$ & The ID of $n e t_{i}$ with $\operatorname{Max}_{c_{i}}$ \\
\hline$a p p_{c_{i}}$ & $\begin{array}{l}\text { The minimum required channel capacity for } \\
\text { each of Video and VoIP applications }\end{array}$ \\
\hline application $_{i_{\text {port }}}$ & $\begin{array}{l}\text { The port number of current } \text { application }_{i} \\
\text { used in transport layer }\end{array}$ \\
\hline Video Port & The port number of Video application \\
\hline VoIP Port $_{\text {P }}$ & The port number of VoIP application \\
\hline$C r_{c_{i}}$ & Cost of current $n e t_{i}$ \\
\hline$C R_{c}$ & Current residual channel capacity of $\mathrm{Cnet}$ \\
\hline idle & determine vehicle $_{i}$ in idle state or not \\
\hline $\bar{i}$ & Counter \\
\hline$t_{\text {AP }}$ idle,$t_{A P_{\text {busy }}}$ & $\begin{array}{l}\text { Time intervals of NAV sittings received via } \\
\text { beacon frame }\end{array}$ \\
\hline$t_{c p}, t_{p i f s}, t_{\text {Ack }}$ pifs & $\begin{array}{l}\text { Time intervals of NAV sittings received via } \\
\text { beacon frame }\end{array}$ \\
\hline$p_{\text {vehicle }}$ & Current position of vehicle $e_{i}$ \\
\hline$p_{A P}$ & Position of the $A P_{i}$ \\
\hline$F S N R_{i}$ & Signal-to-Noise Ratio of $n e t_{i}$ \\
\hline$R_{c_{i}}$ & Residual Channel Capacity of $n e t_{i}$ \\
\hline$t_{c_{i}}$ & Connection Life Time $n^{2} t_{i}$ \\
\hline$C_{i}$ & $\begin{array}{l}\text { The calculated cost by INS scoring function } \\
\text { of each } n e t_{i}\end{array}$ \\
\hline
\end{tabular}

mine the type application $_{i}$. The number of ports that were used for video conferencing and VoIP applications were placed into Video Port and VoIP Port parameters, respectively the first time the of INS algorithm was loaded into the system of each vehicle.

Two main scenarios are presented in this section. In Scenario A, vertical handovers from WLAN to UMTS are examined. In Scenario B, vertical handovers from UMTS to WLAN are investigated. The abbreviations and symbols for the INS Algorithm and the MaxNetworkCost Procedure are presented in Table 1.

\subsection{Scenario (A) Vertical Handover from WLAN to UMTS}

As one of the scenarios used to test handover decision making by the INS algorithm, Scenario A was used to illustrate what happens when a vehicle connected to a WLAN obtains another network candidates that belong to a UMTS $3 \mathrm{G}$ networks. After finding the best network candidate from among the available networks using MaxNetworkCost() procedure, the INS algorithm verified the vehicle's status before processing the handover to the selected network using $\operatorname{Max}_{c_{i}}$. 
The first steps for the INS algorithm to obtain a vertical handover decision included the Video Port, VoIP Port , and the minimum required channel capacity for each of video and VoIP applications using the lookup table $a p p_{c_{i}}$. When the vehicle was in busy-mode the application iport $_{\text {was }}$ wonitored by the INS algorithm as a way to determine if the current application $_{i}$ was a real or non-real time application. When the application $_{i_{p o r t}}$ used by application a $_{i}$ belonged to either the Video Port or VoIP Port, the INS algorithm considered the handover process to be a handover procedure for real-time applications.

During real-time sessions and before a handover decision is made, concerning $M a x_{c_{i}}$, the minimum required $a p p_{c_{i}}$ for the real-time application was compared with the current residual channel capacity $C R_{c}$. If it was less than $C R_{c}$, it indicated that the current WLAN could provide sufficient channel capacity to support the real-time application. In the other words, the vehicle was able to maintain the real-time session with current WLAN AP without performing a handover to the $\operatorname{Max}_{c_{i}}$ network even though that network could provide more channel capacity. The INS in the vehicle decided to keep its point of attachment to the current network (Cnet) and avoid an unnecessary handovers. The INS algorithm insured an uninterrupted real-time session during the busy - mode by suppressing this handover decision because the application could be maintained with the WLAN AP.

When application $_{i_{p o r t}}$ belonged to non-real time applications such as FTP or HTTP, the efficiency (QoS) of wireless network connection was taken into account. In these situations, the INS chose network candidates using $\operatorname{Max}_{c_{i}}$ and initiated the handover but not before the INS algorithm checked if net $t_{I D}$ was a UMTS. If yes, the handover was initiated. When $n t_{I D}$ was a WLAN, the INS confirmed that $V_{i}>$ $50 \mathrm{~km} / \mathrm{h}$. The reason behind this step is that when a network candidate was scored using cost Formula 11 and was identified as a WLAN and velocity of vehicle was $V_{i}>50 \mathrm{~km} / \mathrm{h}$, then the probability of a link connection breakdown will increase due to limited range of coverage [28]. In these cases, handovers to a WLAN were prevented when $V_{i}>50 \mathrm{~km} / \mathrm{h}$ by using the INS algorithm as a way to avoid link connection breakdowns that could negatively affect the QoS for the session during the busy-mode. Handover to WLAN were initiated when $V_{i}<50 \mathrm{~km} / \mathrm{h}$.

\subsection{Scenario (B) Vertical Handover from UMTS to WLAN}

Vertical handover decision making from UMTS $3 \mathrm{G}$ networks to WLANs was illustrated in Scenario B. In this scenario, a vehicle was associated with a UMTS and intended to initiate a vertical handover decision with a WLAN. First, the vehicle's mode was considered. As it in Scenario A, the vehicle's status or mode was defined as being either idle or busy in advance of any handover decisions. Similar to Scenario A, when a vehicle was idle, the INS performed the handover using $\operatorname{Max}_{c_{i}}$.

In busy - mode, before any handover could be performed using $\operatorname{Max}_{c_{i}}$, and if the net $_{I D}$ was a WLAN and the velocity of the vehicle was $V_{i}>50 \mathrm{~km} / \mathrm{h}$, then the vertical handover with WLAN was prevented and the vehicle remained connected to Cnet. However, when $V_{i}<50 \mathrm{~km} / \mathrm{h}$ and net $_{I D}$ was a WLAN, the current application was identified first as being either a real or non-real time application. If the application $_{i_{\text {port }}}$ belonged to either a Video or VoIP application, the vehicle checked if the $\operatorname{app}_{c_{i}}$ was less than UMTS's $B S C R_{c}$. If it was less, the vehicle remained connected to Cnet.

In order to insure the QoS of the real-time session, the INS algorithm also verified that $a p p_{c_{i}}$ was greater than the residual channel capacity $R_{c_{i}}$ of network using $\operatorname{Max}_{c_{i}}$. In this case, INS prevented vertical handovers to WLAN as a way to keep the QoS of the session in the acceptable range. In the other words, since Cnet (UMTS) could provide a channel capacity greater than the elected network using $\operatorname{Max}_{c_{i}}$ (WLAN), the vertical handover to WLAN was not triggered during the real-time application sessions.

For the period of time that a real-time application is running and in addition to a vehicle's velocity, two conditions were verified before a vertical handover to a WLAN. By verifying these conditions, there was no need to perform unnecessary handover decisions to WLAN even if it could provide more channel capacity. Moreover, avoiding vertical handovers to WLAN in these situations, unwanted time delays normally caused by the handover process are thwarted. As a result, the real-time applications were protected from the negative effects of such delays.

Finally, in Scenario B when the application $_{i_{\text {port }}}$ was not related to any of the real-time applications, the INS algorithm performed the handover with the elected network using $\operatorname{Max}_{c_{i}}$ without any conditions. The reason behind this is that during the non-real time session the download speed of the link connection of the associated network was preferred over the continuity of the link connection. Hence, INS algorithm assumed that the network with the $\operatorname{Max}_{c_{i}}$ provided faster connection speeds and directly triggered the handover decision regardless any other network or mobility aspects.

\section{Performance Evaluation}

A performance test minimized handover failures and unnecessary handovers while a vehicle was roaming. Furthermore, to optimize network resources wireless link connection breakdowns were avoided especially those caused by the limited coverage area provided by a WLAN. In this section the performance analyses of the INS scheme in terms 


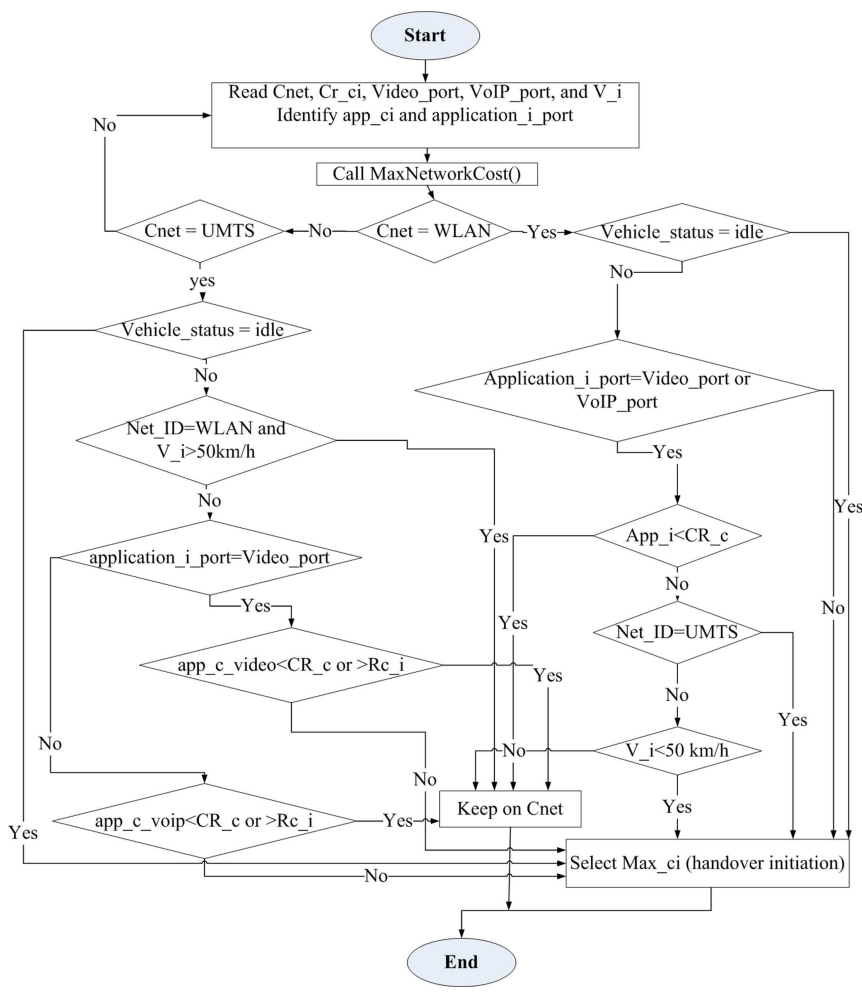

Fig. 7: INS Flow Chart.

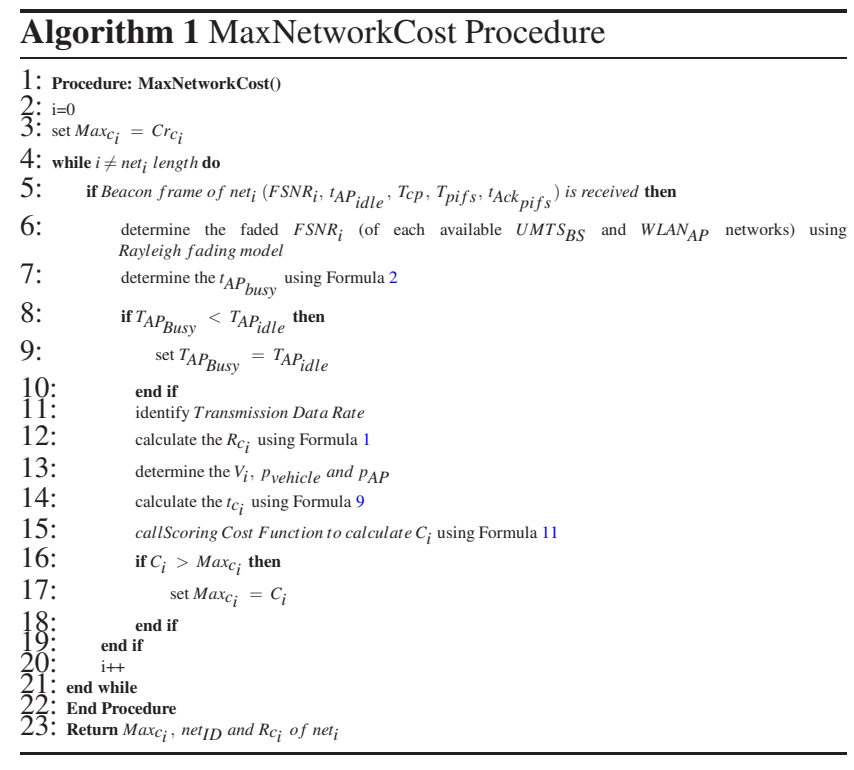

of the probability of unnecessary handovers, link connection breakdowns, and handover failures as well as selected cells and AP IDs, the total average handover delay, packet End-to-End delays and the packet loss ratio for VoIP and Video applications is presented. The proposed INS scheme was compared to multi-criteria utility functions [10] and an integrated scheme [9].

Figure 9 illustrates the performance of the INS scheme and the state of arts at different vehicle velocities. The pro-

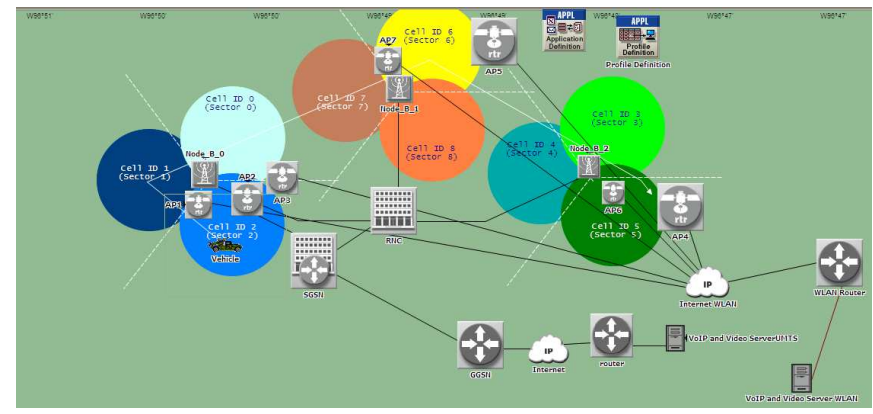

Fig. 8: Simulation Scenario of Vertical handover (WLAN to UMTS) and (UMTS to WLAN) respectively.

posed INS scheme provided excellent performance because it minimized handover failures, unnecessary handovers, and the probability of link connection breakdowns. The reason behind that is the INS scheme efficiently addressed the issues of the network selection process when a vertical handover from UMTS to WLAN was required. This was achieved by precisely identifying the residual channel capacity and link connection life time of each available WLAN candidate. Subsequently, when the velocity of a vehicle increased, the INS scheme performed better by maintaining the probability of unnecessary handovers, link connection breakdowns, and the handover failures.

Figure 10 shows the results of the network selection process that used the INS scheme, Multi-Criteria Utility Function [10], and Integrated Scheme [9]. Figure 10a illustrates the results of selected UMTS's cell ID and WLAN's AP ID when a vehicle is in motion during the scenario presented in Figure 8 for a period of $900 \mathrm{sec}$. The results shown in Figure 10a, were obtained using the proposed INS scheme that was used by each vehicle to select the next network connection link. The important point to note here is that using INS scheme, the vertical handovers were performed only twice in the 163.78(WLAN (AP1) to UMTS (cell1)) and 647.53 (UMTS (cell8) to WLAN (AP4) seconds that the simulations were active.

The Multi-Criteria Utility Function [10] was tested by employing the same simulation scenario. The results from the selected networks during the simulation are illustrated in Figure 10b. According to the graph in Figure 10b, 4 vertical handovers and 1 horizontal handover (between WLAN (AP2) to WLAN (AP1)), were considered to be unnecessary handovers. These handovers were deemed to be unnecessary because after the handover decision was made the vehicle did not spend enough time in the area to connect to the selected APs. The results illustrated in Figure 10b, also reveal that handovers were failed 6 times over the course of the simulation.

An Integrated Scheme [9] was used for comparison purposes and it was exposed to the same simulated scenario. 


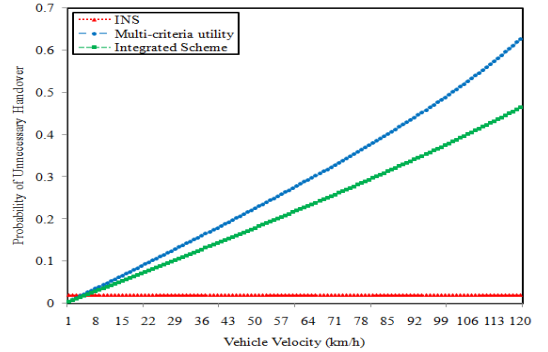

(a) The Unnecessary Handovers Probability.

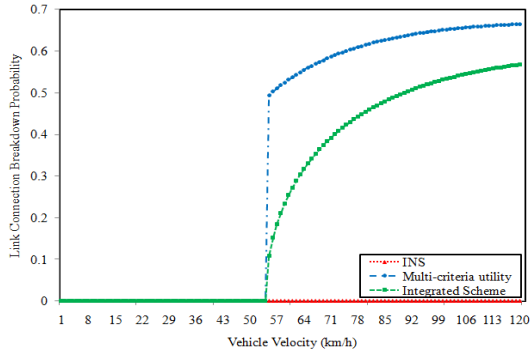

(b) The Link Connection Breakdown Probability.

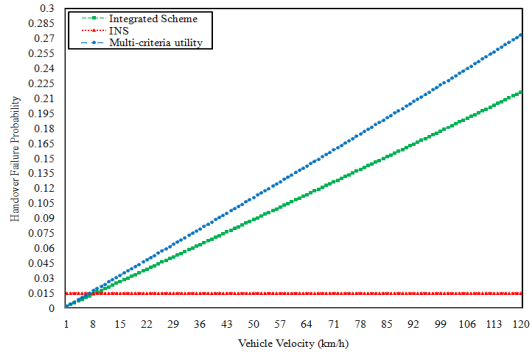

(c) The Handover Failure Probability.

Fig. 9: The performance analyses of INS Scheme in comparison with Utility Function Method and Integrated Scheme vs. Vehicle Velocity.

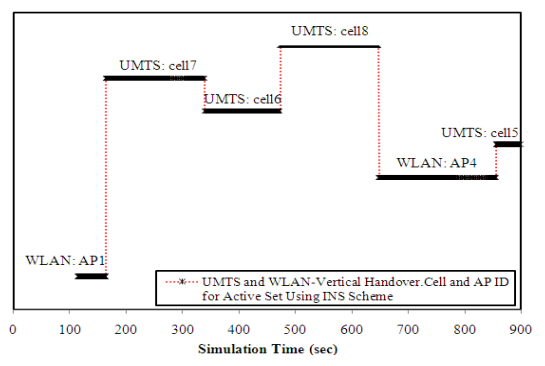

(a) INS Scheme.

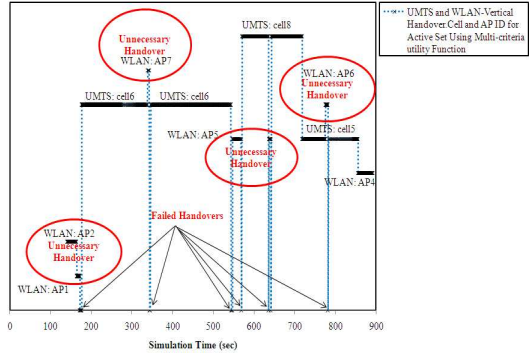

(b) Multi-Criteria Utility Function.

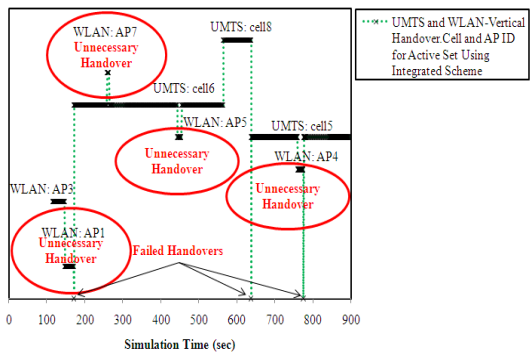

(c) Integrated Scheme.

Fig. 10: Network Selection Results of Selected cell and AP ID vs. Simulation Time.

The network selection results are presented in the Figure 10c. In this figure, there were 3 unnecessary vertical handovers from UMTS to WLAN (AP 7, 5 and 4) and only a single unnecessary horizontal handover between AP3 to AP1.The illustrated results show that the INS scheme efficiently decreased unnecessary vertical handovers that normally occur with WLAN by making an intelligent handover decision by selecting the most suitable network access link.

The total number of handover delays for each of INS scheme, multi-criteria utility function, and the Integrated schem are presented in Figure 11. Figure 11a shows the average handover delay obtained by using the INS scheme in 20 vehicles. The average handover delay was calculated using the number of handover decisions (Vertical or Horizontal) performed by those 20 vehicles as they travelled along the same route in the simulated scenario. The handover delays consisted of several delay periods including link layer handover, movement detection, address allocation, session reconfiguration, and packet re-transmission delays [29]. The results shown in Figure 11a reveal that there were two types of horizontal handovers when the number of handovers increased over the course of the scenario. They were WLANto-WLAN and UMTS-to-UMTS. The average obtained from the handover delays from all 20 vehicles and the WLAN-to-
WLAN and UMTS-to-UMTS using INS scheme were 0.263 and 0.432 seconds, respectively.

On the other hand, the vertical handovers between UMTSto-WLAN had a 0.7 seconds average delay during the fourth handover decision. The handover to WLAN-to-UMTS had an average delay of 0.8 seconds in the fifth handover decision. In this regard, it can be remarked that the time of the vertical handover delay increased when the handover was WLAN-to-UMTS. Based on the results from the implementation and simulation process, the total handover delay for specific handover processes included the delays associated with the establishment and release of a connection. Thus, during WLAN-to-UMTS handovers, the establishment of a connection was higher than the delay experienced during a UMTS-to-WLAN handover process. This is because the delay from the complex radio access bearer and the tunnel setup for UMTS is higher than they are for WLAN [30].

Figure $11 \mathrm{~b}$ demonstrates the average handover delay for 20 vehicles compared to the number of handover decision using multi-criteria utility function [10]. The graph presented in Figure 11b,shows that the vertical handover delay from WLAN-to-UMTS increased sharply with the third handover decision when a multi-criteria utility function was used. An explanation can be found in Figure 10bwhich shows that WLAN:AP7was selected using a multi-criteria utility func- 


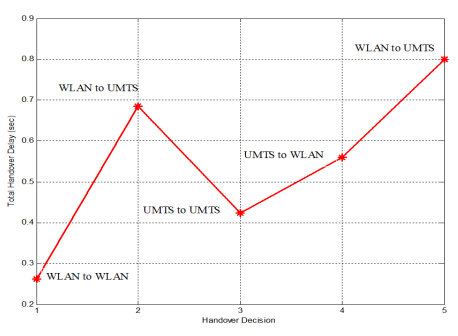

(a) INS Scheme.

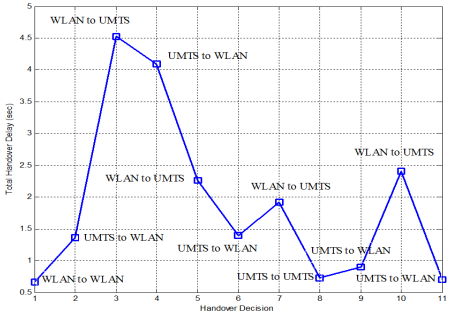

(b) Multi-Criteria Utility Function.

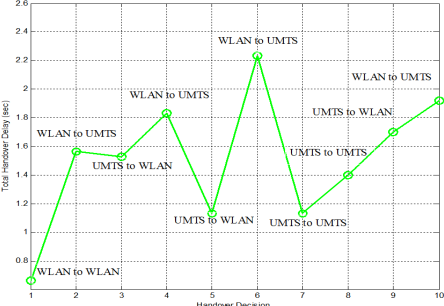

(c) Integrated Scheme.

Fig. 11: The Average Total Handover Delay of 20 Vehicles vs. Handover Decisions.

tion while the vehicle was moving and was considered to be an unnecessary handover. Moreover, during the simulation, handover failures negatively contributed to the total number of handover delays. Afterwards, the forth handover decision was a vertical UMTS-to-WLAN handover with a processing delay of 4.53 seconds. The delay associated with vertical UMTS-to-WLAN handover continued to decrease until it reached 0.7 seconds in the eleventh handover decision that was UMTS-to-WLAN. In contrast, the lowest delay for WLAN-to-UMTS handovers using a multi-criteria utility function was 1.9 seconds, which occurred during the seventh handover decision.

Looking at Figure 11c,reveals the total handover delays presented for the 10 handover decisions using the Integrated Scheme [9]. For the first handover WLAN-to-WLAN decision (between AP3 and AP1), the handover delay was 0.663 seconds. This delay for a horizontal WLAN-to-WLAN handover was associated with handover processes using Integrated Scheme and is the same as the delays experienced while employing a multi-criteria utility function. However, the delay for a horizontal WLAN-to-WLAN handover it is still higher than the delay achieved using our proposed INS scheme.

The delay for WLAN-to-UMTS handover (second handover decision) was 1.527 seconds, which increased to 2.232 seconds when the sixth decision was made. The increase was caused by the vehicle connecting to AP5 which was considered to be an unnecessary handover decision and the connection switched back to UMTS cell6. This re-handoff process with UMTS caused a long delay due to radio delays and tunnel setups. Contrarily, the delays for the WLAN-toUMTS handover decisions decreased to 1.921 seconds for tenth decision. Beforehand, two UMTS-to-UMTS handover decisions were processed with handover delays of 1.132 and 1.4 seconds during the seventh and eighth decisions.

Figures $11 \mathrm{a}, 11 \mathrm{~b}$ and $11 \mathrm{c}$ can illustrate that the INS scheme efficiently compensated for large handover delays associated with handover decisions tackled during the simulations. The success of the INS scheme was due its ability to avoid un- necessary or failed handovers during the simulation, unlike the multi-criteria utility function and Integrated Scheme.

The packet End-to-End delay for real-time applications was evaluated during the simulation to examine the effects of handover processes between different wireless access networks. The performance for VoIP with Codec[G.729A (CSACELP)] was evaluated using the INS scheme, multi-criteria utility functions and Integrated Scheme as illustrated in Figure 12. Figure 12a demonstrates the packet End-to-End delay for VoIP with Codec[G.729A (CS-ACELP)] during the simulation time of $900 \mathrm{sec}$.

The graph presented in Figure 12acompares the VoIP packet End-to-End delays for the INS scheme, the multicriteria utility function, and the Integrated Scheme. By utilizing the INS scheme, the packet End-to-End delay during the VoIP session was kept in the range of 0.14 to 0.16 seconds. By contrast, the End-to-End delay for VoIP using an integrated scheme increased during the simulation until reached it reached a 1 second delay. On the other hand, using a multi-criteria utility function, the End-to-End delay increased sharply to 0.798 seconds in the 126 seconds of simulation time when the VoIP session started. Subsequently, the delay during VoIP session continued to increase when it used the multi-criteria utility function until the delay reached 1.34 seconds.

The packet loss metric for ongoing real-time applications was also investigated in order to illustrate the improvement in performance when our proposed INS scheme was used. During any vertical handover, total packet loss was defined as the aggregation process for all lost packets during vertical handover processes when the vehicle received downlink data packets for ongoing application sessions [31]. Therefore, the average number of handovers during a session contributed to the quantity of packet loss. The packet loss ratio due to vertical handover processes is directly proportionate to the number of handovers during a session.

Figure $12 \mathrm{~b}$ depicts packet loss that occurred during VoIP sessions in normalized form against the number of UMTSto-WLAN handovers processed during the VoIP session. The proposed INS scheme had the lowest packet loss for VoIP 


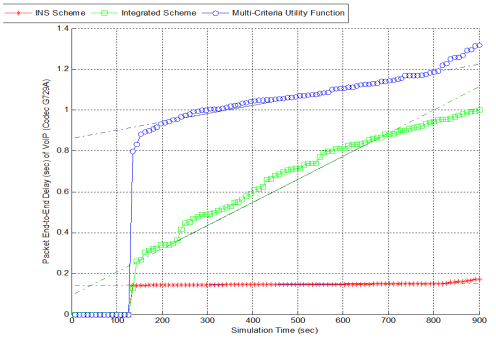

(a) Packet End-to-End Delay vs. Simulation Time.

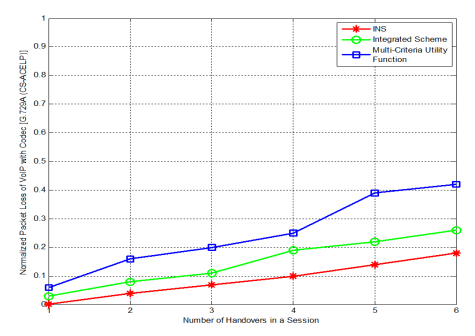

(b) The Normalized Packet Loss Ratio for a UMTS-to-WLAN Handover.

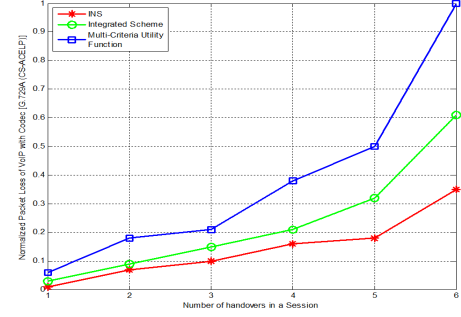

(c) The Normalized Packet Loss Ratio for a WLAN-to-UMTS Handover.

Fig. 12: Performance Evaluation for VoIP Using INS Scheme, Utility Function Method and Integrated Scheme.

applications when the number of handovers increased to 6 UMTS-to-WLAN. The normalized packet loss for the VoIP sessions against the number of handovers from WLAN-toUMTS is presented in Figure 12c. It can be observed that generally the ratio of packet loss increased when the handover was a WLAN-to-UMTS handover. This is because, vertical handover delays occur more often with WLAN-toUMTS handovers than with UMTS-to-WLAN handovers, as it mentioned earlier. Hence, the interruption that normally occurs with wireless communication due to this delay increases as the buffers in the network elements overflow. Nonetheless, the INS scheme performed the best in terms of the lowest packet loss ratio with increasing WLAN-to-UMTS handovers.

A second real-time application examined during the simulated scenario was a video application. An analysis of its performance analyses is illustrated in Figure 13. Figure 13a shows the packet End-to-End delay for a video (MPEG-2 CIF/SIF(625) $352 * 288$ pixels) during the simulation using the three schemes. The End-to-End delay for the video application fluctuated due to the heavy video bit-rates that frequently reached the maximum 30kbps. The packet End-toEnd delays when the proposed INS scheme was used were kept below 1 second during the video session. This delay was considered to be an acceptable delay for video applications [27] and [22]. On the other hand, the multi-criteria utility function and the Integrated Scheme obtained higher packet End-to-End delays when used with the video applications.

Figures $13 \mathrm{~b}$ and $13 \mathrm{c}$ illustrate normalized packet loss ratios and the number of UMTS-to-WLAN and WLAN-toUMTS handovers. Here, the INS scheme achieved the lowest packet loss for both UMTS-to-WLAN and WLAN-toUMTS handovers out of a maximum packet loss of 367 (UMTS-to-WLAN) and 482 (WLAN-to-UMTS).The proposed INS scheme achieved seamless mobility in the VANET by insuring high QoS for real-time applications. The success of the proposed INS scheme was due to its use of a handover decision making mechanism that considered the requirements of real-time applications and the quality of the wireless links. Besides, the intelligent network selection process based on the maximum scoring function of the selected election parameters supports obtaining a vertical handover process with short delays.

\section{Conclusion}

In this paper, an Intelligent Network Selection (INS) scheme -was proposed to make vertical handover decisions in VANET using V2I communications. The proposed INS scheme was designed based on the maximization scoring function to rank available wireless network candidates. The results of the simulations show that the proposed INS scheme outperform existing approaches in terms of decreasing the probability of unnecessary handovers, link connection breakdowns, and handover failures. The proposed INS scheme also decreased average handover delay, packet End-to-End delays for VoIP and Video applications, packet loss ratios for VoIP and Video applications, in addition to making the network selection process more efficient. In the future, extending the proposed INS algorithm by considering different mobility scenarios that can be suitable for various vehicular environments should be undertaken.

\section{References}

1. Y.S. Chen, C.H. Cheng, C.S. Hsu, and G.M. Chiu. Network mobility protocol for vehicular ad hoc networks. In Wireless Communications and Networking Conference., pages 1-6, Budapest, 5-8 April 2009. IEEE.

2. Kayhan Zrar Ghafoor, Kamalrulnizam Abu Bakar, Kevin Lee, and Haidar AL-Hashimi. A novel delay-and reliability-aware intervehicle routing protocol. Network Protocols and Algorithms, 2(2):66-88, 2010.

3. Kayhan Zrar Ghafoor, Kamalrulnizam Abu Bakar, Jaime Lloret, Rashid Hafeez Khokhar, and Kevin C Lee. Intelligent beaconless geographical forwarding for urban vehicular environments. Wireless networks, 19(3):345-362, 2013. 


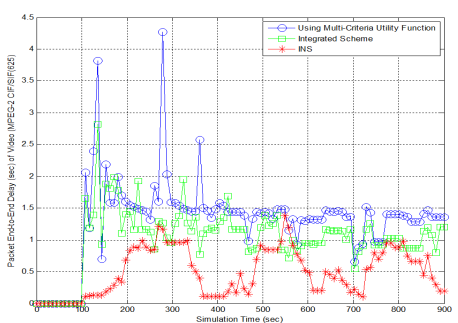

(a) Packet End-to-End Delay vs. Simulation Time.

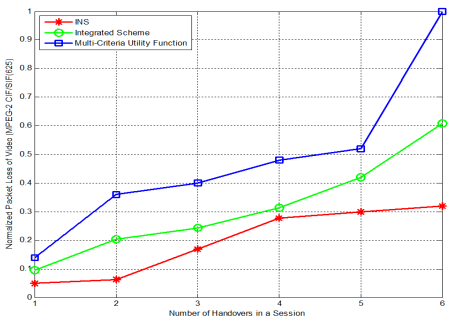

(b) The Normalized Packet Loss Ratio for a UMTS-to-WLAN Handover.

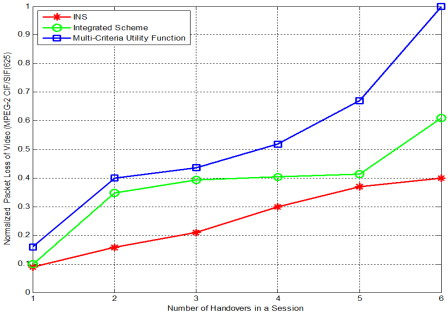

(c) The Normalized Packet Loss Ratio for a WLAN-to-UMTS Handover.

Fig. 13: Performance Evaluation for Video (MPEG-2) Using INS Scheme, Utility Function Method and Integrated Scheme.

4. A. Prakash, S. Tripathi, R. Verma, N. Tyagi, R. Tripathi, and K. Naik. Vehicle assisted cross-layer handover scheme in nemobased vanets (vanemo). International Journal of Internet Protocol Technology, 6(1):83-95, 2011.

5. Cheng-Wei Lee, Meng Chang Chen, and Yeali S Sun. Protocol and architecture supports for network mobility with qos-handover for high-velocity vehicles. Wireless Networks, pages 1-20, 2013.

6. P Pereira, Augusto Casaca, JJPC Rodrigues, VNGJ Soares, Joan Triay, and Cristina Cervelló-Pastor. From delay-tolerant networks to vehicular delay-tolerant networks. Communications Surveys \& Tutorials, IEEE, 14(4):1166 - 1182, 01 September 2011.

7. J. Lloret, A. Canovas, A. Catalá, and M. Garcia. Group-based protocol and mobility model for vanets to offer internet access. Journal of Network and Computer Applications, 36(3):10271038, May 2013.

8. Kayhan Zrar Ghafoor, Jaime Lloret, Kamalrulnizam Abu Bakar, Ali Safa Sadiq, and Sofian Ali Ben Mussa. Beaconing approaches in vehicular ad hoc networks: A survey. Wireless Personal Communications, DOI:10.1007/s11277-013-1222-9, pages $1-28,2013$.

9. L. Wang and G. Kuo. Mathematical modeling for network selection in heterogeneous wireless networksa tutorial. Communications Surveys \& Tutorials, IEEE, (99):1-22, 2011.

10. Q.T. Nguyen-Vuong, Y. Ghamri-Doudane, and N. Agoulmine. On utility models for access network selection in wireless heterogeneous networks. In Network Operations and Management Symposium., pages 144-151, Salvador, Bahia, 7-11 April 2008. IEEE.

11. A. Canovas, D. Bri, S. Sendra, and J. Lloret. Vertical wlan handover algorithm and protocol to improve the iptv qos of the end user. pages 1901 - 1905, Ottawa, ON, 10-15 June 2012.

12. V.K. Varma, S. Ramesh, K.D. Wong, M. Barton, G. Hayward, and J.A. Friedhoffer. Mobility management in integrated umts/wlan networks. In International Conference on Communications, pages 1048-1053, USA, 11-15 May 2003. IEEE

13. S. Mohanty. A new architecture for $3 \mathrm{~g}$ and wlan integration and inter-system handover management. Wireless Networks, 12(6):733-745, 2006.

14. E.J. Rivera-Lara, R. Herrerías-Hernández, J.A. Pérez-Díaz, and C.F. García-Hernández. Analysis of the relationship between qos and snr for an $802.11 \mathrm{~g}$ wlan. In International Conference on Communication Theory, Reliability, and Quality of Service, pages 103-107, Bucharest, Romania, June 29 - July 5 2008. IEEE.

15. Theodore S. Rappaport. wireless communications principles and practice second edition. Prentice Hall, 2002.

16. T. Carpenter. CWNA Certified Wireless Network Administrator Official Study Guide (Exam PWO-100). McGraw-Hill Osborne Media, 2007.

17. J. Eberspacher, J. Eberspächer, C. Bettstetter, H.J. Vögel, C. Hartmann, H.J. Vgel, et al. GSM-Architecture, Protocols and Services. Wiley, 2009.
18. WLAN-MAC. Wireless lan medium access control (mac) and physical layer specifications. IEEE Computer Society, 2007. http://standards.ieee.org/getieee802/802.11.html.

19. C. Kappler. UMTS networks and beyond. Wiley, 2009.

20. K. Egoh and S. De. A multi-criteria receiver-side relay election approach in wireless ad hoc networks. In Military Communications Conference., pages 1-7, Washington, DC, 23-25 Oct 2006. IEEE.

21. P. Bucciol, F. Ridolfo, and J.C. De Martin. Multicast voice transmission over vehicular ad hoc networks: issues and challenges. In Seventh International Conference on Networking, pages 746-751, Cancun, 13-18 April 2008. IEEE.

22. G. Thonet, P. Allard-Jacquin, and P. Colle. Zigbee-wifi coexistence. Schneider Electric White Paper, 2008.

23. A.F. Ribadeneira. An analysis of the mos under conditions of delay, jitter and packet loss and an analysis of the impact of introducing piggybacking and reed solomon fec for voip. Computer Science Theses, page 44, 2007.

24. S. Karapantazis and F.N. Pavlidou. Voip: A comprehensive survey on a promising technology. Computer Networks, 53(12):20502090, 2009.

25. C. Ortiz, J.F. Frigon, B. Sanso, and A. Girard. Effective bandwidth evaluation for voip applications in ieee 802.11 networks. In Wireless Communications and Mobile Computing Conference., pages 926-931, Crete Island, 6-8 Aug 2008. IEEE.

26. International Telecommunication Union. Telecommunication Standardization Sector. Methods for subjective determination of transmission quality. International Telecommunication Union, 1996.

27. D. Li and J. Pan. Evaluating mpeg-4/avc video streaming over ieee 802.11 wireless distribution system. In Wireless Communications and Networking Conference, 2008. WCNC 2008. IEEE, pages 2147-2152. IEEE, 2008.

28. X. Yan, N. Mani, and YA Cekercioglu. A traveling distance prediction based method to minimize unnecessary handovers from cellular networks to wlans. Communications Letters, IEEE, 12(1):14-16, 2008.

29. S.C. Lo, G. Lee, W.T. Chen, and J.C. Liu. Architecture for mobility and qos support in all-ip wireless networks. Selected Areas in Communications, IEEE Journal on, 22(4):691-705, 2004.

30. Rastin Pries, Dirk Staehle, Phuoc Tran-Gia, and Thorsten Gutbrod. A seamless vertical handover approach. In Lloren CerdAlabern, editor, Wireless Systems and Mobility in Next Generation Internet, 5122, pages 167-184. Springer Berlin Heidelberg, 2008.

31. K.S. Munasinghe and A. Jamalipour. An analytical evaluation of mobility management in integrated wlan-umts networks. Computers \& Electrical Engineering, 36(4):735-751, 2010. 